\title{
THERMOREGULATION THROUGH SKIN AT LOW ATMOSPHERIC TEMPERATURES
}

\author{
D. B. Gurung \\ Department of Natural Science (Mathematics), \\ School of Science, Kathmandu University \\ P.O. BOX NO. 6250, Kathmandu, NEPAL. \\ Corresponding author: db_gurung01@yahoo.com; db_gurung@ku.edu.np \\ Received 17 October, 2008; Revised 9 December, 2008
}

\begin{abstract}
The metabolic heat generation decreases exponentially if the persistence of cooling in human body is sustained. This phenomena is under consideration in dermis and subcutaneous tissue to study the exact solution of temperature distribution in dermal layers at low atmospheric temperatures. Other suitable variable physiological conditions are taken and the solution has been obtained using laplace tranform in one dimensional case.
\end{abstract}

Key words: Thermoregulation, Human dermal part, Laplace transform.

2000 Mathematics Subject Classification: 92 C 35.

\section{INTRODUCTION}

Human body maintains core temperature almost exactly constant within a small range $\left( \pm 0.6^{\circ} \mathrm{C}\right.$ or $\left.\pm 1^{\circ} \mathrm{F}\right)$ despite large fluctuations in atmosphric temperatures except when a person develops febrile illness (Guyton and Hall, 2004). The skin and subcutaneous tissue (SST) region is the major organ that controls heat flow to and from the surrounding environment. So the skin temperature, in contrast to the core temperature rises and falls with the temperature of surroundings.

The skin mainly consists of two layers - epidermis and dermis. Epidermis is the most outer part of human skin. It is nonvascular and lined by keratinized stratified squamous epithelium with distint cell types and cell layers. The epidermis contains no blood vessels and hence nutrients and waste products diffuse through the dermo-epidermal layer in order to maintain tissue integraty. The dermis is composed of connective tissue layer and it binds the dermis to epidermis. The population density of blood vessels in the dermis is very thin near the interface of epidermis and dermis but increases gradually becoming almost uniform in the subcutaneous part. This help to understand the variation of physical and physiological parameters like rate of metabolic heat generation, rate of blood mass flow and thermal conductivity of tissue in the layer of SST region with regard to position. 
In the present study, the skin surface is assumed to expose to the environment and the outer skin surface temperature is considered to be equal to the atmospheric temperature, $T_{a}$. The rate of mass blood flow in dermis and subcutaneous tissue is assumed to be position dependent but negligible in epidermis. Further the rate of metabolic heat generation is assumed to be negligible in epidermis but decreasing exponentially in dermis and subcutaneous tissue. The thermal conductivity is taken as constant but different in three layers.

\section{MATHEMATICAL FORMULATION}

The general mathematical model for heat flow in dermal layers is given by

$$
\rho c \frac{\partial T}{\partial t}=\nabla \cdot(K \nabla T)+M\left(T_{b}-T\right)+S
$$

where $M=m_{b} c_{b} ; m_{b}=$ rate of mass blood flow; $c_{b}=$ blood specific heat; $\rho=$ tissue density; $c=$ tissue specific heat; $K=$ tissue thermal conductivity; $T_{b}=$ body core temperature $\left(37^{\circ} \mathrm{C}\right) ; S=$ rate of metabolic heat generation.

Perl [1962] and Cooper and Trezek [1972] have used the equation (1) to study the solution of some simple problems of infinite tissue media assuming all the parameters as constant. Chao et al. [1973] and Chao and Yang [1975] applied finite difference method to solve steady and unsteady models with all physiological parameters as constants. Saxena and his co-workers (Saxena and Bindra, 1987; Saxena et al., 2007; saxena and Gupta, 1994) have used the above equation extensively for the investigation of thermoregulation at normal and abnormal atmospheric temperatures using finite element method. Saxena and Singh [2004] have used laplace transform for temperature distribution in SST region at normal atmospheric temperature.

Here we consider the thickness of SST region in one dimensional case is measured from outer skin surface towards body core. The thickness of epidermis, dermis and subcutaneous tissue have been considered as $a_{1}, a_{2}-a_{1}$ and $a_{3}-a_{2}$ respectively.

The biological structure of human dermal part makes it reasonable to consider $M$ and $S$ zero in epidermis. Accordingly, we consider the biophysics and physiological parameters for each layer of SST region in the following manner:

(i) For epidermis $\left(0<x<a_{1}\right)$

$$
K=K_{1} ; M=M_{1}=0 ; S=S_{1}=0
$$

(ii) For dermis $\left(a_{1}<x<a_{2}\right)$

$$
\begin{aligned}
& K=K_{2} \\
& M=M_{2}=\left(\frac{x-a_{1}}{a_{2}-a_{1}}\right) m \\
& S=S_{2}=\left(\frac{x-a_{1}}{a_{2}-a_{1}}\right) S_{3}
\end{aligned}
$$


(iii) For subcutaneous tissue $\left(a_{2}<x<a_{3}\right)$

$$
\begin{aligned}
K & =K_{3} ; M=M_{3}=m ; \\
S & =S_{3}=s_{0} e^{-\nu t}, \quad \nu \geq 0
\end{aligned}
$$

\section{SOLUTION OF THE PROBLEM}

If the cooling in the human body is sustained, then the tissue cells loss water. This causes the rate of metabolic heat generation decrease exponentially and is given by the relation

$$
S=s_{0} e^{-\nu t}, \quad \nu \geq 0
$$

where $s_{0}$ is the normal metabolic activity and has different value at different skin layers, while $\nu$ is the control parameter which differs from person to person and from one part of the body to another part.

The partial differential equation (1) in one dimensional unsteady state case is

$$
\rho c \frac{\partial T}{\partial t}=K\left(\frac{\partial^{2} T}{\partial x^{2}}\right)+M\left(T_{b}-T\right)+S
$$

Using $S$ from equation (3) in equation (4), and then taking laplace transform with respect to $t$ on both sides of (4), we get

$$
K \frac{d^{2} \bar{T}(x, s)}{d x^{2}}+M\left(\frac{T_{b}}{s}-\bar{T}(x, s)\right)+\frac{s_{0}}{s+\nu}=\rho c s \bar{T}(x, s)-\rho c T(x, 0)
$$

where $\bar{T}(x, s)$ is the laplace transform of $T(x, t)$.

Initially when blood enters in the body tissue (i.e., time zero) the temperature of SST region is assumed to be same as the blood temperature because no heat loss take place at time zero. That is

$$
T(x, 0)=T_{b}
$$

Using equation (6) in equation (5), we get

$$
\frac{d^{2} \bar{T}}{d x^{2}}-\alpha^{2} \bar{T}=\beta
$$

where $\alpha=\sqrt{\frac{M+\rho c s}{K}} ; \beta=-\frac{1}{k}\left[\frac{s_{0}}{s+\nu}+\frac{(M+\rho c s) T_{b}}{s}\right]$

The equation (7) is a non-homogeneous second order linear differential equation. So its general solution is

$$
\bar{T}(x, s)=A e^{\alpha x}+B e^{-\alpha x}+W
$$

where $W=\frac{s_{0}}{M-\rho c \nu}\left[\frac{1}{s+\nu}-\frac{1}{s+M / \rho c}\right]$ and $A$ and $B$ are constants to be determined. For this, we consider the following boundary conditions: 
(i) At outer skin surface:

$T(x=0, t)=T_{a} \Rightarrow \bar{T}(0, s)=T_{a} / s$

(ii) At inner core:

$$
T\left(a_{3}, t\right)=T_{b} \Rightarrow \bar{T}\left(a_{3}, s\right)=T_{b} / s
$$

Applying the boundary conditions in equation (8), we get

$$
\begin{aligned}
\bar{T}(x, s) & =\left(T_{b}-T_{a}\right) \frac{\sin h \alpha\left(x-a_{3}\right)}{s \sin h \alpha a_{3}}+\left(\frac{s_{0}}{M-\rho c \nu}\right)\left[\frac{\sin h \alpha\left(x-a_{3}\right)}{(s+\nu) \sin h \alpha a_{3}}-\frac{\sin h \alpha x}{(s+\nu) \sin h \alpha a_{3}}\right] \\
& +\left(\frac{s_{0}}{M-\rho c \nu}\right)\left[\frac{\sin h \alpha x}{(s+M / \rho c) \sin h \alpha a_{3}}-\frac{\sin h \alpha\left(x-a_{3}\right)}{(s+M / \rho c) \sin h \alpha a_{3}}\right] \\
& +\left(\frac{s_{0}}{M-\rho c \nu}\right)\left[\frac{1}{s+\nu}-\frac{1}{s+M / \rho c}\right]+\frac{T_{b}}{s}
\end{aligned}
$$

Now taking inverse laplace transformation on both sides of equation (9), we obtain

$$
\begin{aligned}
T(x, t) & =\left(T_{b}-T_{a}\right)\left[\frac{\sin h \sqrt{M / K}\left(x-a_{3}\right)}{\sin h \sqrt{M / K} a_{3}}-2 \pi K \sum_{n=1}^{\infty} \frac{n(-1)^{n} e^{-\left(\frac{M}{\rho c}+\frac{n^{2} K \pi^{2}}{\rho c a_{3}^{2}}\right) t} \sin \left(\frac{n \pi\left(x-a_{3}\right)}{a_{3}}\right)}{M a_{3}^{2}+n^{2} K \pi^{2}}\right] \\
& +\left(\frac{s_{0} e^{-\nu t}}{M-\rho c \nu}\right)\left[\frac{\sin h \sqrt{M / \rho c-\nu} \sqrt{\rho c / K}\left(x-a_{3}\right)}{\sin h \sqrt{M / \rho c-\nu} \sqrt{\rho c / K} a_{3}}-\frac{\sin h \sqrt{M / \rho c-\nu} \sqrt{\rho c / K} x}{\sin h \sqrt{M / \rho c-\nu} \sqrt{\rho c / K} a_{3}}\right] \\
& +\left(\frac{2 s_{0} \pi K}{M-\rho c \nu}\right)\left[\sum_{n=1}^{\infty} \frac{n(-1)^{n} e^{-\left(\frac{M}{\rho c}+\frac{n^{2} \pi^{2}}{\rho c a_{3}^{2}}\right) t}}{M a_{3}^{2}+n^{2} K \pi^{2}-\nu \rho c a_{3}^{2}}\left\{\sin \left(\frac{n \pi x}{a_{3}}\right)-\sin \left(\frac{n \pi\left(x-a_{3}\right)}{a_{3}}\right)\right\}\right] \\
& +\left(\frac{2 s_{0}}{(M-\rho c \nu) \pi}\right)\left[\sum_{n=1}^{\infty} \frac{(-1)^{n} e^{-\left(\frac{M}{\rho c}+\frac{n^{2} K \pi^{2}}{\rho c a_{3}^{2}}\right) t}}{n}\left\{\sin \left(\frac{n \pi\left(x-a_{3}\right)}{a_{3}}\right)-\sin \left(\frac{n \pi x}{a_{3}}\right)\right\}\right] \\
& +\frac{s_{0} e^{-\nu t}}{M-\rho c \nu}+T_{b}
\end{aligned}
$$

The equation (10) gives the temperature distribution in different layers of skin and subcutaneous tissue region.

\section{NUMERICAL RESULTS AND DISCUSSION}

The value of control parameter $\nu$ depends on the value of $s_{0}$, the degree of low temperature and the duration of exposure to cold source. The suitable value of $\nu$ is found to be 0.30 when the skin surface is exposed to cold source for a time of 20 minutes for $s_{0}=0.0357$ 
$\mathrm{cal} / \mathrm{cm}^{3}$-min. The following value of physical and physiological constants have been taken prescribed by (Cooper and Trezek, 1972; Saxena, 1983) to obtain the numerical results.

$$
\begin{aligned}
K_{1} & =0.060 \mathrm{cal} / \mathrm{cm}-\min ^{\circ} \mathrm{C} \\
K_{2} & =0.045 \mathrm{cal} / \mathrm{cm}-\min ^{\circ} \mathrm{C} \\
K_{3} & =0.030 \mathrm{cal} / \mathrm{cm}-\min ^{\circ} \mathrm{C} \\
\rho & =1.05 \mathrm{~g} / \mathrm{cm}^{3} \\
c & =0.83 \mathrm{cal} / \mathrm{g} \\
m & =0.003 \mathrm{cal} / \mathrm{cm}^{3}-\min ^{\circ} \mathrm{C}
\end{aligned}
$$

The thickness of dermal layers have been taken as:

$$
\begin{aligned}
a_{1} & =0.10 \mathrm{~cm} \\
a_{2}-a_{1} & =0.30 \mathrm{~cm} \\
a_{3}-a_{2} & =0.50 \mathrm{~cm}
\end{aligned}
$$

It is assumed that the skin has been exposed to cold sources of constant temperature $2^{\circ} \mathrm{C}$ and $-2^{\circ} \mathrm{C}$ for a time of 20 minutes. Accordingly we observed the graphs of tissue temperatures at different times within 20 minuts as shown in figures 1 to 4 . We also observed the graphs of tissue temperatures at different layers of SST region for these two source temperatures at the time of 10 minutes as shown in figures 5 to 6 .

As the temperature changes, the thermoreceptor present in the skin send information to the hypothalamus centers which in turn controls the rate of blood mass flow and rate of metabolic heat generation. At low temperature for example below $15^{\circ} \mathrm{C}$, the blood vessels constrict causing low blood mass flow rate and increase in the rate of metabolic heat generation rate to regulate the body core temperature at low atmospheric temperatures. But if the persistence of cooling is sustained, then the metabolic heat generation rate decreases exponentially due to the loss of water in cells and mass of blood flow rate behaves periodic.

From the figures 1 to 4, we observe that SST region temperature changes very rapidly for the first few minutes and then afterwards become steady. This steady temperature is higher for higher atmospheric temperature. This is due to the reason that as temperature decreases (below $15^{\circ} \mathrm{C}$ ) the rate of constriction of blood vessels increases causing the decrease of blood flow in blood vessels and hence decreases SST temperature. The other reason is that the metabolic heat generation rate decreases exponentially if the body is continually exposed at constant cold source. Figures 5 and 6 exhibit the same phenomena. The figures also explain that tissue temperature increases from skin surface toward body core. This is because the concentration of blood vessels are negligible in epidermis and increases from the interface of epidermis and dermis toward the interface of dermis and subcutaneous tissue with uniformaly distributed in subcutaneous tissue.

\section{ACKNOWLEDGEMENT}

The author acknowledges Prof. V. P. Saxena for his valuable suggestions. 


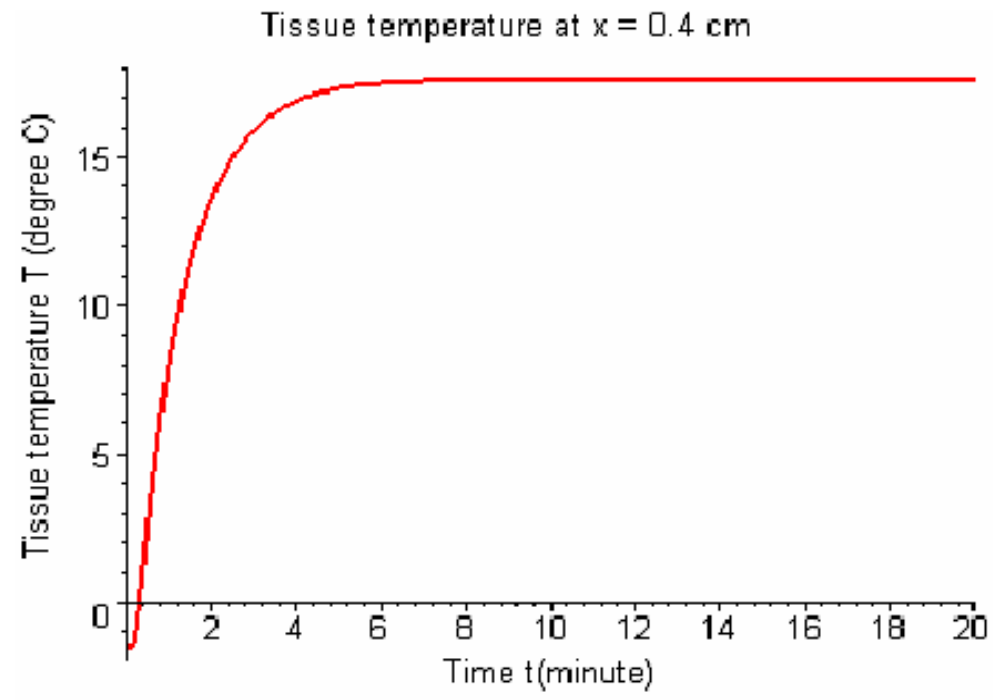

Figure 1: Tissue temperature of dermal part for $T_{a}=2^{\circ} \mathrm{C}$.

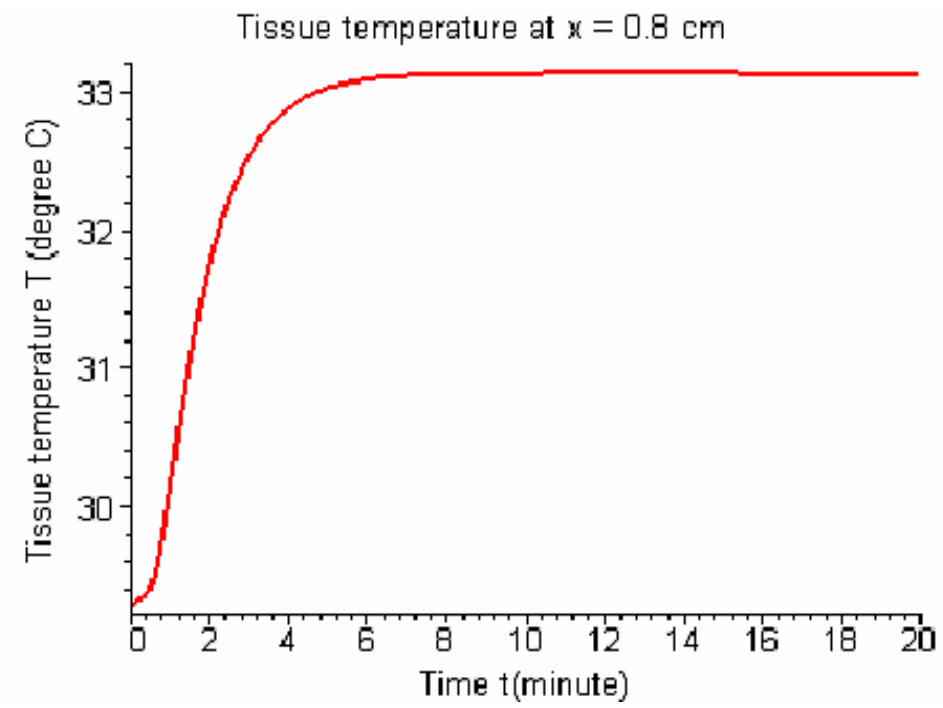

Figure 2: Tissue temperature of dermal part for $T_{a}=2^{\circ} \mathrm{C}$. 


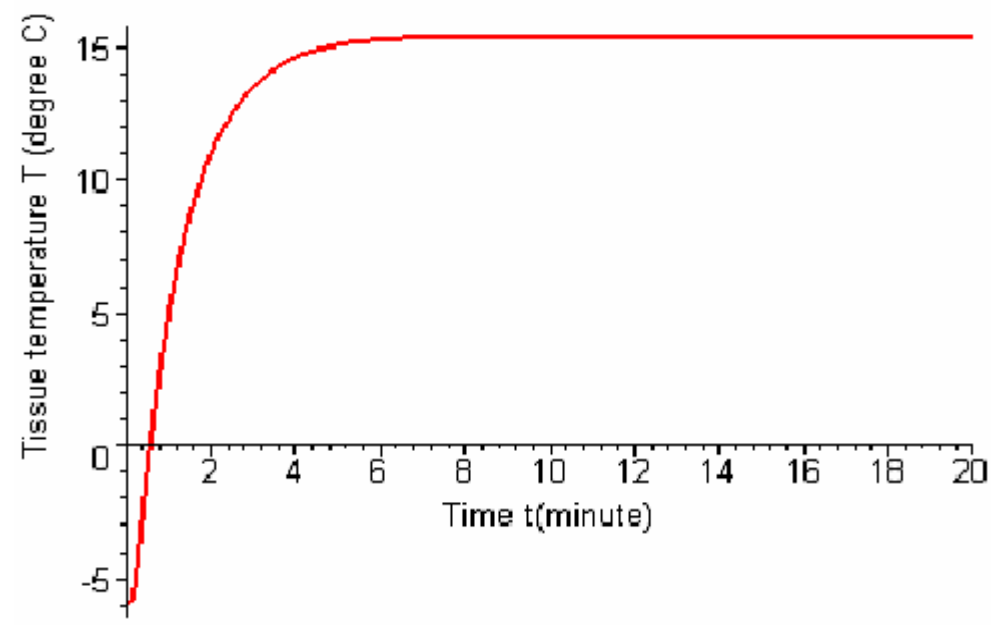

Figure 3: Tissue temperature of dermal part for $T_{a}=-2^{\circ} \mathrm{C}$.

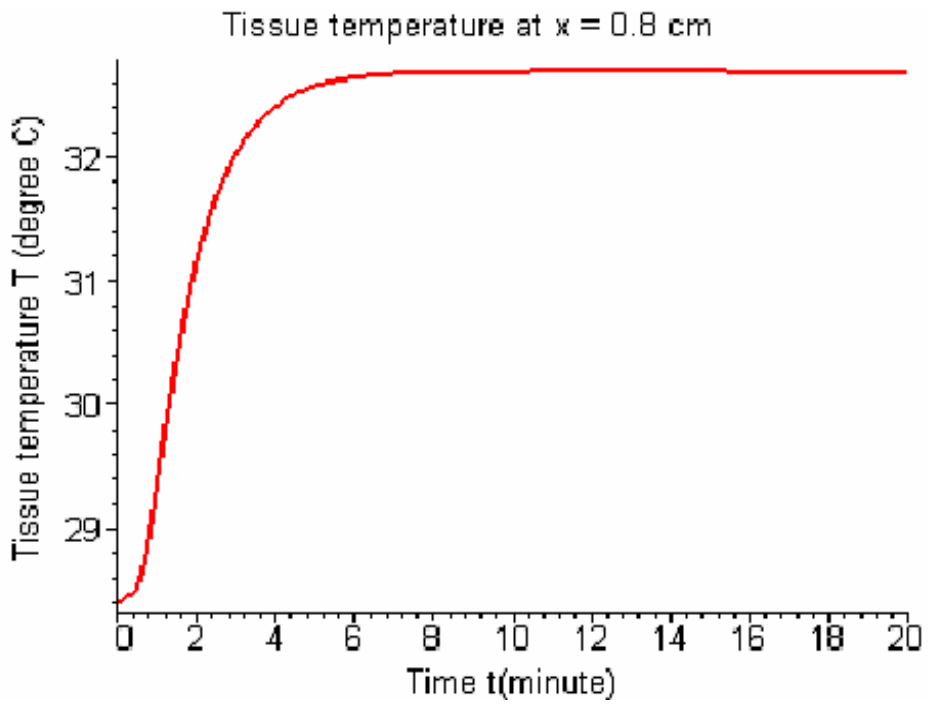

Figure 4: Tissue temperature of dermal part for $T_{a}=-2^{\circ} \mathrm{C}$. 


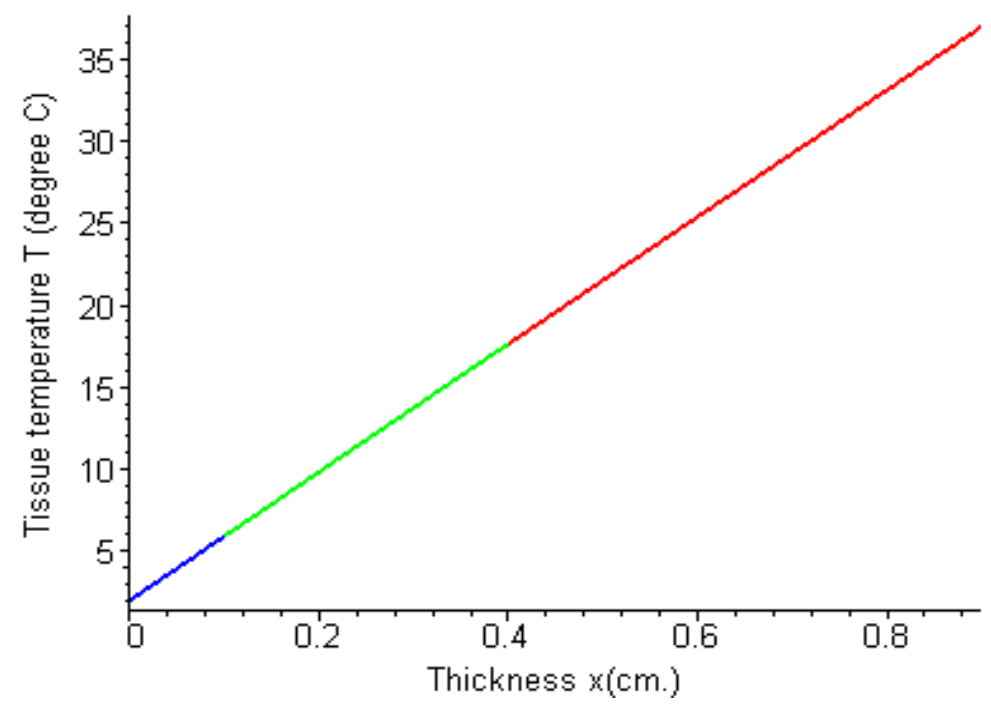

Figure 5: Tissue temperature of dermal part at $T_{a}=2^{\circ} \mathrm{C}$ at time 10 minutes.

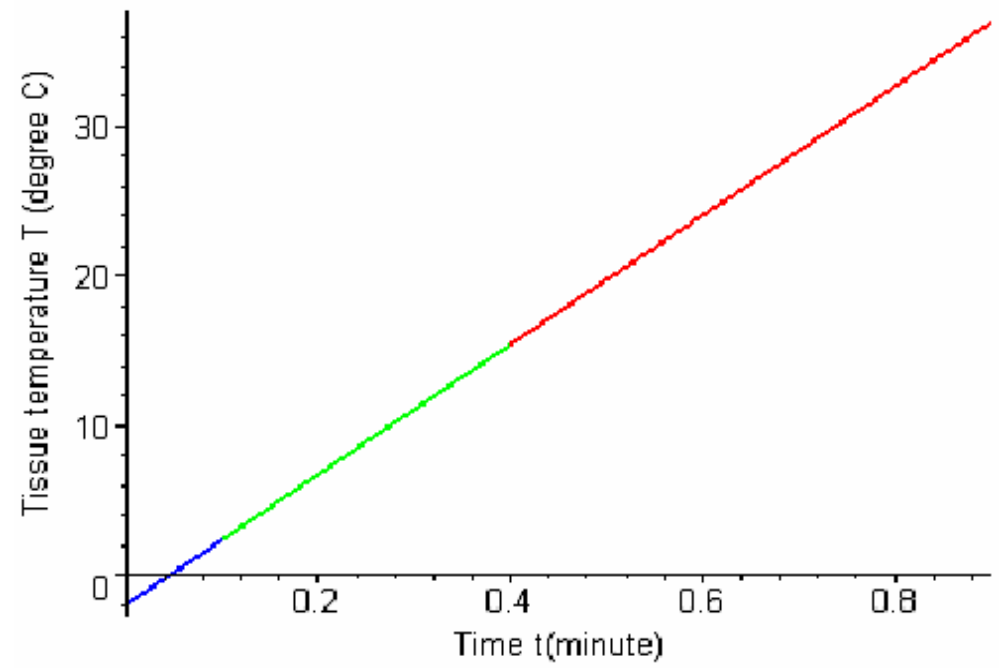

Figure 6: Tissue temperature of dermal part at $T_{a}=-2^{\circ} \mathrm{C}$ at time 10 minutes. 


\section{REFERENCES}

1. Chao K. N., Eisley. J. G. and Yang W. J., Heat and water migration in the regional skin and subcutaneous tissue, Bio. Mech. Sympo. ASME, 1973, 69-72.

2. Chao K. N. and Yang W. J., Response of skin and tissue in sauna and steam baths, Bio. Mech. Sympo. ASME, 1975, 69-71.

3. Cooper T. E. and Trezek G. J., A probe technique for determining the thermal conductivity of tissue, J. Heat Transfer, ASME, 94,1972, 133-140.

4. Guyton A. C. and Hall J. E., Text book of medical physiology, Tenth edition, Indian reprint, W. B. Saunders, 2004.

5. Myers G. E., Analytic methods in conduction heat transfer, Book CO. Inc., New York, Mc. Graw Hill, 1971, 320-362.

6. Patterson A. M., Measurement of temperature profiles in human skin, South African J.Sci. 72, 1976, 78-79.

7. Perl W., Heat and matter distribution in body tissues and the determination of tissue blood flow by local clearance method, J.Theor. Biolo. 2,1962, 201-235.

8. Saxena V. P., Temperature distribution in human skin and subdermal tissues, J. Theor. Biol. 102, 1983, 277-286.

9. Saxena V. P. and Bindra J. S., Quadratic shape functions in variational finite element approach to heat distribution in cutaneous and subcutaneous tissues, Indian J.Pure. Appl. Math. 18(9), 1987, 846-855.

10. Saxena V. P., Adhikary P. R. and Gurung D. B., Quadratic shape function FEM approach to temperature distribution problem in peripheral layers of human body, Bulletin of the Allahabad Mathematical Society 22, 2007, 21-36.

11. Saxena V. P., Adhikary P. R. and Gurung D. B., Mathematical estimation of unsteady state burn damage due to hot temperature, The Nepali Math. Sc. Report, 27(1\&2), 2007, 75-84

12. Saxena V. P., Adhikary P. R. and Gurung D. B., Mathematical study of heat regulation in human dermal parts with variable heterogeneity, Proc. Nat. Acad. Sci. India, 77(A), IV, 2007, 334-337.

13. Saxena V. P. and Gupta M. P., Variational finite element approach to a heat flow problem in human limbs, Internat. J. Math. \& Math. Sci. 17(4), 1994, 771-778.

14. Saxena V. P. and Singh R., Unsteady state radial heat flow in the outer layers of spherical regions of human or animal body, 34, 2004, 47-54. 